\title{
Argumentieren und Erzählen in der politischen Kommunikation
}

\author{
Juliane Schröter $\cdot$ Constanze Spieß
}

Angenommen: 16. März 2021 / Online publiziert: 6. April 2021

(C) Der/die Autor(en) 2021

Argumentieren und Erzählen sind grundlegende sprachlich-kommunikative Praktiken, die uns fast ständig und überall begegnen. Dementsprechend weitreichend und vielfältig sind die Forschungstraditionen zum Argumentieren und zum Erzählen. Auffällig ist allerdings, dass sich diese Forschungstraditionen - gesamthaft betrachtet und von Ausnahmen abgesehen - bis in die jüngste Zeit weitgehend unabhängig voneinander entwickelt haben. ${ }^{1}$ Dies spiegelt sich u.a. in den Disziplinen, die derzeit eine vorrangige Zuständigkeit für das Argumentieren oder aber das Erzählen beanspruchen. Sind dies für das Argumentieren vor allem die Linguistik, die Argumentationstheorie bzw. argumentation studies sowie auch die Logik bzw. Philosophie, sind es im Falle des Erzählens primär die Literaturwissenschaft, die Medien- und Filmwissenschaft, aber ebenso sozialwissenschaftliche Disziplinen wie etwa die Soziologie. Die bis heute andauernde relative Unverbundenheit der Forschung kommt gleichermaßen darin zum Ausdruck, dass Argumentieren bzw. Argumentation einerseits und Erzählen bzw. Narration andererseits zwar nicht immer, aber oft unter

\footnotetext{
${ }^{1}$ Für die Situation ist bezeichnend, dass das Register des einschlägigen »Handbook of argumentation theory« weder narration noch narrative noch story telling als Stichwort enthält. Das vorhandene Stichwort zu »narrativity« führt zu einem kurzen Absatz über eine einzige Publikation aus den 1980er Jahren (vgl. Eemeren/Garssen/Krabbe u.a. 2014, S. 984, 442). Dementsprechend findet sich auch im Register des umfangreichen »Handbook of narratology« (Hühn/Pier/Schmid u.a. (Hg.) 2009, S. 455) kein Lemma argument oder argumentation.
}

\section{J. Schröter}

Département de langue et de littérature allemandes, Université de Genève, Genf, Schweiz

E-Mail: juliane.schroeter@unige.ch

C. Spieß $(\bowtie)$

Institut für Germanistische Sprachwissenschaft, AG Pragmalinguistik, Philipps-Universität Marburg, Marburg, Deutschland

E-Mail: constanze.spiess@uni-marburg.de 
unterschiedliche Oberbegriffe gefasst werden. Beispielsweise wird »Argumentation« als »complex speech act« (Eemeren/Grootendorst 1992, S. 10) oder - nicht unzutreffend - als »Typus sprachl.[ichen] Handelns, dessen genauer theoret.[ischer] Status noch immer umstritten ist « (Ehlich 2016, S. 56), bezeichnet, während »Narration« bzw. »Erzählung « außer als Gattung z.B. als »Diskursmuster« (Hoffmann 2018, S. 218) oder »[t]extuelles Ordnungsmuster« (Zeman 2016, S. 185) gilt.

Zwar gibt es keine allgemein akzeptierten Bestimmungen von Erzählen und Argumentieren, doch scheint ein relativ großer Konsens darüber zu bestehen, dass eine Erzählung eine »Geschehensdarstellung « zur Voraussetzung hat, d.h. dass eine Erzählung »singuläre und konkrete Gegenstände und Sachverhalte« in einer »Sequenz chronologisch geordneter Ereignisse« darstellt, die »auch räumlich, zeitlich oder kausal« verbunden sind (Martínez 2017, S. 2). Eine verhältnismäßig große Übereinstimmung gibt es offenbar auch darüber, dass eine Argumentation aus »at least one argumentative inference or argument composed of one or more premises and one conclusion (of which at least one premise is explicit) « besteht und zudem »as an attempt to overcome or reduce either a difference of standpoints or doubts regarding the correctness of a standpoint « fungiert (Schröter 2019, S. 298).

Aus diesen Bestimmungen lassen sich verschiedene, strukturelle Gemeinsamkeiten des Argumentierens und Erzählens ableiten, die es ermöglichen, sowohl das Argumentieren als auch das Erzählen als sprachlich-kommunikative Praktik aufzufassen (zu diesem Vorschlag vgl. Spieß/Tophinke 2018, S. 193, Schröter 2019, S. 298). So handelt es sich beim Argumentieren wie beim Erzählen zunächst einmal um Typen menschlichen Handelns mit unterschiedlichen Funktionen. Beide Typen werden vorrangig geschrieben- und gesprochensprachlich realisiert, sind aber keineswegs auf diese Modalitäten beschränkt, d.h. sie können durch Bilder, Gestik, Mimik usw. unterstützt und ergänzt werden. Argumentieren und Erzählen sind zudem beide in ihrer Komplexität kaum festgelegt, sie können also in einfacher Form, auf wenig Raum und in wenig Zeit, wie auch in stark ausdifferenzierter Form, über Stunden oder Hunderte von Seiten hinweg entfaltet werden. Argumentieren und Erzählen können andere Praktiken in sich integrieren und selbst Teil von anderen, übergreifenden Praktiken sein. Argumentieren und Erzählen sind darüber hinaus beide - z.B. mit Blick auf das, was sie als common sense voraussetzen bzw. etablieren deutlich kulturell geprägt wie auch kulturell prägend.

Die genannten Gemeinsamkeiten legen es nahe, Argumentieren und Erzählen systematisch zusammenzudenken und ihre Beziehung zueinander genauer zu beleuchten (zum bestehenden Forschungsbedarf in diesem Bereich vgl. Carranza 2015, S. 74 sowie Hannken-Illjes 2018, S. 153, 163, und 2019, S. 47, die auch verschiedene Ansätze vorstellt, Narration und Argumentation aufeinander zu beziehen). Dabei wird rasch deutlich, dass Argumentieren und Erzählen in einem Verhältnis der potenziellen gegenseitigen Inklusion und Konstitution stehen. Gemeint ist damit, dass häufig argumentiert wird, indem (u.a.) erzählt wird, und dass oft erzählt wird, indem (u.a.) argumentiert wird. Argumentationen können sogar zentral durch Narrationen gestützt sein und aus diesen hervorgehen, wie auch Narrationen wesentlich aus Argumentationen bestehen und aus diesen heraus entwickelt werden können. Darauf ist in verschiedenen Publikationen, die aus ganz verschiedenen Disziplinen und Sprachräumen hervorgegangen sind, in den letzten ca. 15 Jahren zunehmend auf- 
merksam gemacht worden (vgl. z.B. Danblon et al. (Hg.) 2008 oder Olmos (Hg.) 2017).

Ein Bereich, in dem sich dieses Wechselverhältnis von Argumentieren und Erzählen in besonders eindrücklicher Weise zeigt, ist die politische Kommunikation: Behördenkampagnen bedienen sich des sogenannten >strategischen Storytellings<, Kandidierende für politische Ämter begründen ihre Eignung dafür mit biographischen Anekdoten und Erzählungen, politische Talkshows führen gesellschaftliche Problemlagen mithilfe von Betroffenen vor, die ihre Erlebnisse erzählen, Parteien nutzen narrative Comics zur Wahlwerbung, und in Gedenkreden werden historische Großnarrative für Schlüsse auf die politische Gegenwart aktualisiert; des Weiteren integrieren Werke zur politischen Geschichte Argumentationen in ihre Erzählung von Schlüsselereignissen, Memoiren von Politiker*innen legitimieren politische Entscheidungen nachträglich mit Argumenten, und bei Pressekonferenzen versuchen Anwesende, strittige Erzählungen von Geschehnissen argumentativ zu plausibilisieren - das Spektrum der Verbindungen von Erzählen und Argumentieren im Bereich der politischen Kommunikation ist also offensichtlich von überwältigender Breite und scheint sich derzeit insbesondere in internetbasierten Kommunikationsformen noch zusätzlich zu erweitern.

Im Kontrast zu dieser Fülle der Verbindungen von Argumentieren und Erzählen in der politischen Kommunikation steht die bisherige Beschäftigung der Politolinguistik damit: Während das Interesse an der Beschäftigung mit narrativen Strukturen innerhalb der politischen Kommunikation in der politikwissenschaftlichen sowie kommunikations- und medienwissenschaftlichen Forschung in jüngster Zeit gewachsen ist (vgl. Jarzebski 2013, Gadinger/Jarzebski/Yildiz (Hg.) 2014, Müller 2020 etc.), muss die Beschäftigung mit politischen Narrationen und Narrativen seitens der Politolinguistik generell als eher marginal bezeichnet werden. Das ist umso erstaunlicher, als die Politolinguistik auf die Ergebnisse der linguistischen Erzählforschung zurückgreifen könnte, die gerade für die Erforschung nicht-fiktionaler, nicht-literarischer Erzählungen hilfreiche Analysekonzepte und -methoden entwickelt hat (vgl. u.a. Spieß/Tophinke 2018). Die Auseinandersetzung mit Argumenten und Argumentationen hingegen ist in der Politolinguistik fest etabliert. Hier fällt allerdings auf, dass neuere theoretische Ansätze aus den internationalen argumentation studies bisher eher zögerlich rezipiert worden sind (so etwa die pragmadialectical argumentation theory, vgl. z.B. Eemeren/Grootendorst 2004, oder das argumentum model of topics, vgl. u.a. Rigotti/Greco 2019). Entsprechend der bisherigen politolinguistischen Erforschung des Erzählens einerseits und des Argumentierens andererseits ist auch das Verhältnis bzw. die Verbindung von Argumentieren und Erzählen in der politischen Kommunikation bisher kein Schwerpunkt der empirischen und theoretischen Politolinguistik geworden (für Ausnahmen vgl. aber z.B. Weidacher 2018 oder Girnth/Burggraf 2019a und 2019b).

An dieser Stelle setzt das vorliegende Themenheft an. Es steht in einer Linie mit einem jüngeren Themenheft der Zeitschrift für Literaturwissenschaft und Linguistik (LiLi), das sich unter dem Titel »Narration - Persuasion - Argumentation « dem Zusammenhang von Narration und Argumentation im Allgemeinen widmet (vgl. Hannken-Illjes/Till/Bleumer (Hg.) 2019), sowie mit einem weiteren Heft, das unter dem Titel »Alltagspraktiken des Erzählens« verschiedene Formen des nicht-li- 
terarischen Erzählens erschließt (vgl. Spieß/Tophinke (Hg.) 2018). Das vorliegende Themenheft fokussiert aber im Gegensatz dazu das Wechselverhältnis von Argumentieren und Erzählen speziell in der politischen Kommunikation. Sein Ziel ist es, herauszuarbeiten, wie die Praktik des Erzählens als Teil des politischen Argumentierens fungieren kann und wie umgekehrt die Praktik des Argumentierens als Element des politischen Erzählens genutzt werden kann. Dazu enthält das Heft verschiedene Analysen empirischer Ausschnitte der politischen Kommunikation, wobei in allen Studien des Heftes ausgehend vom jeweils analysierten empirischen Material auch theoretisch reflektiert wird, in welcher Form und mit welchen Funktionen Argumentieren und Erzählen in der politischen Kommunikation verbunden werden können.

In den Beiträgen zeigt sich, dass enge Verbindungen von Erzählen und Argumentieren in den klassischen und in neueren Kommunikationsformen und Medien der politischen Kommunikation zu finden sind: in Reden im Parlament (im Beitrag von Spieß) ebenso wie in Nachrichten im Fernsehen (im Beitrag von Luginbühl), in Leserbriefen in der Zeitung (im Beitrag von Schröter) genauso wie in User-Kommentaren (im Beitrag von Michel/Pfurtscheller) und in verschwörungstheoretischen Videos im Internet (in den Beiträgen von Römer und Niehr). In der Zusammenschau der Beiträge fällt auf, wie vielfältig das Zusammenspiel von Narration und Argumentation ist: So finden sich z.B. Erzählungen, die als Argument für einen Standpunkt fungieren (etwa in Parlamentsreden, Leserbriefen oder User-Kommentaren), aber auch Argumente, die als Teil einer Erzählung vorgebracht werden (etwa in Verschwörungstheorien oder, zutreffender, Verschwörungsgeschichten). Weiterhin zeigen sich komplexe Kommunikate, die punktuell Argumentationen und Narrationen enthalten, aber auch zugleich als Ganze als Argumentation oder Narration betrachtet werden können (so Beiträge zu Fernsehnachrichten, die O-Töne und andere Elemente enthalten) oder gar Serien von Einzelerzählungen, die als Gesamtmenge ein Argument ergeben (so User-Kommentaren im Internet).

In den Beiträgen zeichnen sich einige weiterführende Fragen ab, die im Rahmen dieses Themenheftes nicht beantwortet werden konnten, deren Beantwortung aber als lohnenswert erscheint: So deutet sich in einigen Beiträgen an, dass es kulturelle bzw. sprachliche und nationale Unterschiede in der Art und Weise gibt, erzählend zu argumentieren bzw. argumentierend zu erzählen. Welche Rolle spielen solche kulturellen Unterschiede bei der Verbindung der beiden Praktiken? Deutlich wird auch, dass die Verbindung von Argumentieren und Erzählen keine ahistorische ist, sondern sich im Laufe der Zeit verändert. Nimmt die Verbindung der beiden Praktiken im Laufe der Zeit zu? Lassen sich historische Phasen ausmachen, in denen sie eher verbunden bzw. eher getrennt realisiert werden? Schließlich stellt sich im Überblick über die Beiträge die Frage, ob und inwieweit die Verbindung von Erzählen und Argumentieren in bestimmten Bereichen der politischen Kommunikation häufiger zu beobachten ist als in anderen. Ist die Verbindung z.B. in der Kommunikation mit potenziellen Wählenden, einem breiten Publikum bzw. der allgemeinen Bevölkerung häufiger als in der Kommunikation, die sich ausschließlich an Politiker*innen richtet? Und falls das so wäre, auf welche (möglichweise stereotypen) Vorstellungen der politischen Akteur*innen von den Wirkungen des Argumentierens und Erzählens ließe dies schließen? 
Funding Open Access funding enabled and organized by Projekt DEAL.

Open Access Dieser Artikel wird unter der Creative Commons Namensnennung 4.0 International Lizenz veröffentlicht, welche die Nutzung, Vervielfältigung, Bearbeitung, Verbreitung und Wiedergabe in jeglichem Medium und Format erlaubt, sofern Sie den/die ursprünglichen Autor(en) und die Quelle ordnungsgemäß nennen, einen Link zur Creative Commons Lizenz beifügen und angeben, ob Änderungen vorgenommen wurden.

Die in diesem Artikel enthaltenen Bilder und sonstiges Drittmaterial unterliegen ebenfalls der genannten Creative Commons Lizenz, sofern sich aus der Abbildungslegende nichts anderes ergibt. Sofern das betreffende Material nicht unter der genannten Creative Commons Lizenz steht und die betreffende Handlung nicht nach gesetzlichen Vorschriften erlaubt ist, ist für die oben aufgeführten Weiterverwendungen des Materials die Einwilligung des jeweiligen Rechteinhabers einzuholen.

Weitere Details zur Lizenz entnehmen Sie bitte der Lizenzinformation auf http://creativecommons.org/ licenses/by/4.0/deed.de.

\section{Literatur}

Carranza, Isolde E. (2015): Narrating and arguing. From plausibility to local moves. In: Anna De Fina/Alexandra Georgakopoulou (Hg.): The Handbook of narrative analysis. Chichester: Blackwell, S. 57-75.

Danblon, Emmanuelle/De Jonge, Emmanuel/Kissina, Ekaterina u.a. (Hg.) (2008): Argumentation et narration. Bruxelles: Editions de l'Université de Bruxelles.

Eemeren, Frans H. van/Grootendorst, Rob (1992): Argumentation, communication, and fallacies. A pragma-dialectical perspective. Hillsdale: Erlbaum.

Eemeren, Frans H. van/Grootendorst, Rob (2004): A systematic theory of argumentation. The pragmadialectical approach. Cambridge: Cambridge Univ. press.

Eemeren, Frans H. van/Garssen, Bart/Krabbe, Erik C.W. u.a. (2014): Handbook of argumentation theory. Dordrecht: Springer.

Ehlich, Konrad ( ${ }^{5}$ 2016): Argumentation. In: Helmut Glück/Michael Rödel (Hg.): Metzler-Lexikon Sprache. 5., aktual. und überarb. Aufl. Stuttgart: Metzler, S. 56.

Gadinger, Frank/Jarzebski, Sebastian/Yildiz, Taylan (Hg.) (2014): Politische Narrative. Konzepte - Analysen - Forschungspraxis. Wiesbaden: Springer. DOI: https://doi.org/10.1007/978-3-658-02581-6.

Girnth, Heiko/Burggraf, Stefan (2019a): Narration und Persuasion in der politischen Rede. In: LiLi. Zeitschrift für Literaturwissenschaft und Linguistik 49 (1), S. 107-119.

Girnth, Heiko/Burggraf, Stefan (2019b): Narrative Überzeugungs- und Wirkungsmechanismen der politischen Rede. In: Armin Burkhardt (Hg.): Handbuch Politische Rhetorik. Berlin: de Gruyter, S. $565-582$.

Hannken-Illjes, Kati (2018): Argumentation. Einführung in die Theorie und Analyse der Argumentation. Tübingen: Narr Francke Attempto.

Hannken-Illjes, Kati (2019): »Siehe da, es gab Ermessensspielräume«. Argumentatives Erzählen - Erzählendes Argumentieren. In: LiLi. Zeitschrift für Literaturwissenschaft und Linguistik 49 (1), S. 29-49.

Hannken-Illjes, Kati/Till, Dietmar/Bleumer, Hartmut (Hg.) (2019): Narration - Persuasion - Argumentation. Themenheft. LiLi. Zeitschrift für Literaturwissenschaft und Linguistik 49 (1).

Hoffmann, Ludger (2018): Erzählen aus funktional-pragmatischer Perspektive. In: LiLi. Zeitschrift für Literaturwissenschaft und Linguistik 48 (2), S. 203-224.

Hühn, Peter/Pier, John/Schmid, Wolf u.a. (Hg.) (2009): Handbook of narratology. Berlin: de Gruyter.

Jarzebski, Sebastian (2013): Wahlkampf als Erzählung. Metaphern und Narrative im TV-Duell. In: KarlRudolf Korte (Hg.): Die Bundestagswahl 2013. Wiesbaden: Springer, S. 367-385. DOI: https://doi. org/10.1007/978-3-658-02915-9_16.

Martínez, Matías (2017): Was ist Erzählen? In: Matías Martínez (Hg.): Erzählen. Ein interdisziplinäres Handbuch. Stuttgart: Metzler, S. 2-6.

Müller, Michael (2020): Politisches Storytelling. Wie Politik aus Geschichten gemacht wird. Köln: Halem. Olmos, Paula (Hg.) (2017): Narration as argument. Cham: Springer. 
Rigotti, Eddo/Greco, Sara (2019): Inference in argumentation. A topics-based approach to argument schemes. Cham: Springer.

Schröter, Juliane (2019): The TV addresses of the Swiss government before popular votes. A case study of argumentation in direct democracy. In: Journal of argumentation in context 8 (3), S. 285-316.

Spieß, Constanze/Tophinke, Doris (2018): Alltagspraktiken des Erzählens. In: LiLi. Zeitschrift für Literaturwissenschaft und Linguistik 48 (2), S. 193-201, https://doi.org/10.1007/s41244-018-0089-3.

Spieß, Constanze/Tophinke, Doris (Hg.) (2018): Alltagspraktiken des Erzählens. Zeitschrift für Literaturwissenschaft und Linguistik 48(2).

Weidacher, Georg (2018): Erzählen als Element politischer Kommunikation in Sozialen Medien. In: LiLi. Zeitschrift für Literaturwissenschaft und Linguistik 48 (2), S. 309-330.

Zeman, Sonja ( $\left.{ }^{5} 2016\right)$ : Erzählung. In: Helmut Glück/Michael Rödel (Hg.): Metzler-Lexikon Sprache. 5., aktual. und überarb. Aufl. Stuttgart: Metzler, S. 185. 